\title{
Quantum estimation of coupling strengths in driven-dissipative optomechanics
}

\author{
Kamila Sala, ${ }^{1,2}$ Anton Doicin, ${ }^{2}$ Andrew D. Armour, ${ }^{1,3}$ and Tommaso Tufarellii ${ }^{1,2}$ \\ ${ }^{1}$ Centre for the Mathematics and Theoretical Physics of Quantum Non-Equilibrium Systems, \\ University of Nottingham, Nottingham, NG7 2RD, United Kingdom \\ ${ }^{2}$ School of Mathematical Sciences, University of Nottingham, Nottingham, NG7 2RD, United Kingdom \\ ${ }^{3}$ School of Physics and Astronomy, University of Nottingham, Nottingham, NG7 2RD, United Kingdom
}

We exploit local quantum estimation theory to investigate the measurement of linear $\left(g_{1}\right)$ and quadratic $\left(g_{2}\right)$ coupling strengths in a driven-dissipative optomechanical system in the red-sideband regime. We consider model parameters inspired by recent experiments, in the regime $g_{2}<g_{1}$. We find that: (i) $g_{1}$ is easier to estimate than $g_{2}$ at lower driving strengths, while (ii) strong enough driving allows the two parameters to be estimated with similar relative precision. Our analysis also reveals that the majority of information about $g_{1}$ and $g_{2}$ is encoded in the reduced state of the mechanical element, and that the best estimation strategy for both coupling parameters is well approximated by a direct measurement of the mechanical position quadrature. Interestingly, we also show that temperature does not always have a detrimental effect on the estimation precision.

\section{INTRODUCTION}

Quantum optomechanics focuses on the interaction between the electromagnetic radiation and motional degrees of freedom of mechanical oscillators [1]3. The simplest optomechanical system consists of a single cavity mode interacting with a single mechanical mode and is realised, for example, in an optical cavity with a movable mirror. In this case the mechanism responsible for the interaction is radiation pressure, which entails momentum exchange between light and matter. The presence of a cavity boosts the otherwise weak radiation pressure force, enhancing the light-matter interaction. The quantum effects of radiation pressure forces and the associated limits they set on the precision of mirrordisplacement measurements are of great importance for many applications including gravitational wave detectors, scanning probe microscopy and force sensing [1 4].

Although the radiation pressure interaction is intrinsically non-linear [5], approximate models are usually used [1, 2] which assume a linear dependence of the cavity frequency $\omega\left(X_{b}\right)$ on the dimensionless position of the movable mirror, $X_{b}$. So far these "linear" models have proved extremely successful, aided by the fact that the bare (or 'singlephoton') optomechanical coupling strength is usually very small [1, 2]. However, researchers are continuously exploring ways of enhancing the optomechanical coupling, as well as the potential of optomechanics for ultra-high-accuracy applications such as Planck physics [6]. Hence, extensions to the linear model are becoming a necessity. The next step beyond the linear approach is to expand the cavity frequency up to and including second order in $X_{b}$, lead-

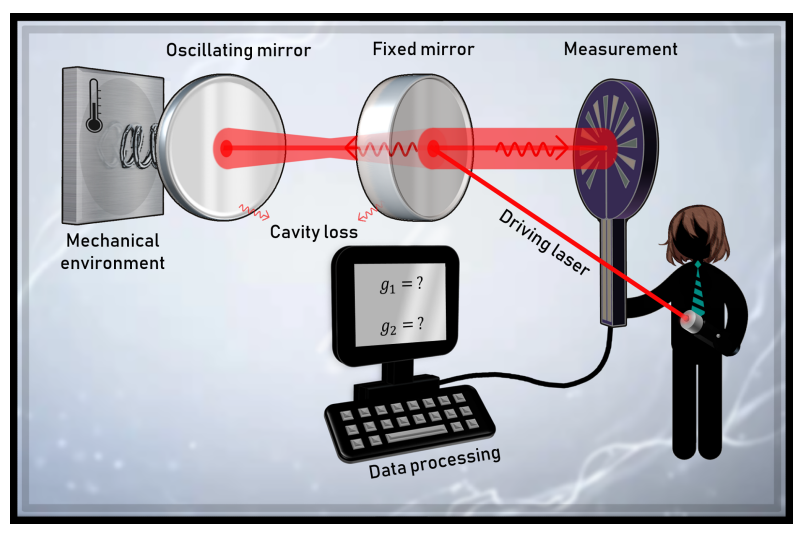

FIG. 1: Schematic of the parameter estimation methodology for driven-dissipative optomechanics. We consider a driven-dissipative optomechanical system featuring a driven and lossy cavity, where one of the mirrors is also a damped mechanical oscillator. The mechanical support has low but finite temperature. To carry out the estimation procedure we wait for the system to reach a steady state, then measure an observable. We repeat the process (relaxation to steady state plus measurement) many times to get reliable statistics. Finally, we process the data to find the best guess for the coupling parameters of interest.

ing to what we will call the "quadratic model" [7, 8]. Accurate knowledge of all the relevant optomechanical coupling parameters will indeed be crucial for virtually any application of these systems. With such motivation in mind, this paper exploits local quantum estimation theory [9] (QET) to investigate how precisely the linear and quadratic coupling strengths may be measured in a model quantum optomechanical system. In a nutshell, QET looks for the best strategy for estimating unknown parameters encoded in the density matrix of a quantum 
system (i.e. a quantum statistical model) [10, 11]. The ultimate limits to the precision with which the desired parameters can be estimated may be quantified via the quantum Cramér-Rao bounds 9, 12, 13.

Within such framework, we consider an optomechanical set-up featuring a driven (and lossy) cavity, whose dynamics is described by a Lindblad master equation. As is typical in recent optomechanics experiments, we assume sufficiently strong driving to approximate the dynamics via a master equation that is bilinear in the canonical operators. This leads to a Gaussian steady state [14, whose first and second moments we characterise via a combination of matrix algebra and numerical methods. This state, with its explicit dependence on all the model parameters (in particular the unknown coupling strengths), will embody our quantum statistical model. This in turn may be attacked via general closed-form expressions that are available for QET in Gaussian models [15. The visual representation of the parameter estimation methodology for driven-dissipative optomechanics is shown in Fig. 1.

Using this approach, and for parameters inspired by recent experimental works, we find that at low intracavity photon numbers the linear coupling strength is easier to estimate than the quadratic one. At high intracavity photon numbers, however, the achievable estimation precisions for the two constants are comparable.

We then investigate how well some specific measurements perform when compared to the fundamental limits imposed by QET. We in particular focus on measurements of the mechanical position $X_{b}$, field amplitude $Q$, mechanical momentum $P_{b}$ and field phase $P$, which can all be analyzed within the Gaussian formalism [16]. In the range of intracavity photon numbers explored, we find that the best strategy for estimating the coupling parameters is through a direct measurement of the mechanical position $X_{b}$.

We additionally explored the influence of temperature on the estimation precision of the coupling strengths. In the case of the linear coupling parameter we found that the effect of temperature is mostly significant at lower intracavity photon numbers (i.e. lower driving strengths), where it improves the estimation precision. At higher intracavity photon numbers, the zero temperature scenario predicts a better estimation precision instead. Interestingly, in the case of the quadratic coupling parameter we found that a hotter mechanical bath gives a higher estimation precision at all intracavity photon numbers in our range.

We note that the application of QET in closely related optomechanical set-ups was previously con- sidered in works by Bernad, Sanavio and Xuereb [11, [17, who adopted the linear model of optomechanics. In Ref. [11, purely Hamiltonian (non-dissipative) dynamics was assumed, and it was found that larger intracavity photon numbers would facilitate the estimation of the linear coupling strength. Analogously, our results to follow show that a similar conclusion holds in the strong driving regime. However, we also find that for weaker drive strengths the picture is more complicated when considering finitetemperature effects. We note that very recently the same authors went on to consider a driven-damped system [17] though using a somewhat different approach to ours and without considering quadratic couplings. In particular, Ref. 17] neglects the contribution of the steady state's first moments (i.e. the averages of the canonical operators) to the quantum Fisher information (QFI). We checked that this is a well-justified assumption for the model parameters adopted therein. However, our results show clearly that there are experimentally accessible parameter regimes where the picture changes dramatically and the first moments can come to dominate the QFI. Finally, Schneiter et al. [18] have applied local QET to a time-dependent, purely Hamiltonian and quadratic optomechanical system. There, a nontrivial dependence of the QFI (and hence the precision bounds of the coupling parameters) on the temperature was also observed.

This paper is organised as follows. In Sec. II] we introduce our model of driven-dissipative optomechanics, including both linear and quadratic coupling terms, and outline how the dynamics may be approximated via a bilinear master equation in the limit of strong driving. In Sec. III we calculate the steady state of the system, which is Gaussian within the considered approximations, and hence is fully characterized by its first and second moments. We then develop the necessary QET tools to investigate the optimal estimation of linear and quadratic coupling parameters. In Sec. IV] we present and discuss the findings of our research. Finally, in Sec. $\mathrm{V}$ we summarise our results.

\section{MODEL}

We consider a simple optomechanical system consisting of two quantum harmonic oscillators, describing a single-mode cavity field and a single mechanical mode, respectively. The two modes are coupled non-linearly via radiation pressure 1 1 3 . We thus assume that our system is described by the Hamil- 
tonian

$$
H_{0}=\hbar \omega\left(\hat{X}_{b}\right) \frac{\hat{Q}^{2}+\hat{P}^{2}}{2}+\hbar \omega_{m} \frac{\hat{X}_{b}^{2}+\hat{P}_{b}^{2}}{2},
$$

with $\hat{Q}$ and $\hat{P}$ the amplitude and phase quadratures for the cavity mode, $\hat{X}_{b}$ and $\hat{P}_{b}$ the dimensionless position and momentum operators of the movable mirror, whilst $m$ and $\omega_{m}$ are the effective mass and frequency of the mechanics [5, 7, 11, 19]. The only nontrivial commutators read $[\hat{Q}, \hat{P}]=\left[\hat{X}_{b}, \hat{P}_{b}\right]=i$. The optomechanical coupling arises from a parametric dependence of the cavity frequency on the mechanical position $\omega\left(\hat{X}_{b}\right)$. We note that in this work we rely heavily on the formalism of Gaussian states [16, hence we find it convenient to model our system via dimensionless quadrature operators. These are denoted via capital letters to avoid confusion with the commonly used dimensional operators of the mechanics.

In the widely accepted "linear" model of optomechanics, the mechanical range of motion is assumed to be very small (e.g. compared to the average cavity length) and $\omega\left(\hat{X}_{b}\right)$ is approximated by an expansion to linear order in $\hat{X}_{b}$. For the "quadratic model" of optomechanics, instead, terms up to and including $\hat{X}_{b}^{2}$ are retained:

$$
\omega\left(\hat{X}_{b}\right) \approx \omega_{0}+\omega^{\prime}(0) \hat{X}_{b}+\frac{1}{2} \omega^{\prime \prime}(0) \hat{X}_{b}^{2},
$$

where $\omega_{0}$ is the bare cavity frequency [1, 2, 7]. The strength of the optomechanical interaction can be quantified with the linear and quadratic coupling strengths, which for a generic set-up are defined as

$$
\begin{aligned}
& g_{1} \equiv \frac{1}{\sqrt{2}} \omega^{\prime}(0), \\
& g_{2} \equiv \frac{1}{2} \omega^{\prime \prime}(0),
\end{aligned}
$$

respectively [2]. Note that we can always ensure that $g_{1}$ is positive by a redefinition of the positive direction of $X_{b}$, and that the linear model is recovered by setting $g_{2}$ to zero.

A purely Hamiltonian description of the system is however not sufficient for our purposes, since we aim to describe a (more realistic) driven-dissipative optomechanical system featuring a driven and lossy cavity, as well as a damped mechanical oscillator in contact with a thermal bath. In order to conveniently introduce coherent driving in the model, we shall move to a frame rotating at the frequency of the driving laser, $\omega_{L}$. In this frame the Hamiltonian of the driven system may be written as

$$
\begin{aligned}
H= & \hbar\left(\Delta_{0}+\omega^{\prime}(0) \hat{X}_{b}+\frac{1}{2} \omega^{\prime \prime}(0) \hat{X}_{b}^{2}\right) \frac{\hat{Q}^{2}+\hat{P}^{2}}{2} \\
& +\hbar \omega_{m} \frac{\hat{X}_{b}^{2}+\hat{P}_{b}^{2}}{2}+\sqrt{2} \hbar \mathcal{E} \hat{Q},
\end{aligned}
$$

where $\Delta_{0}=\omega_{0}-\omega_{L}$ is the detuning between the cavity and driving laser and $\mathcal{E}$ is the drive amplitude.

In our model we will include weak cavity decay at a rate $\kappa \ll \omega_{0}$ and weak mechanical damping at a rate $\Gamma_{m} \ll \omega_{m}$, assuming that the thermal occupation of the cavity mode is negligible. We assume that the corresponding master equation describing the dynamics of the system is of the general Lindblad form [20 22]:

$$
\begin{aligned}
\dot{\rho}(t)= & -\frac{i}{\hbar}[H, \rho(t)] \\
& +\sum_{i j} \frac{\gamma_{i j}}{2}\left[2 \hat{R}_{i} \rho(t) \hat{R}_{j}-\left\{\hat{R}_{j} \hat{R}_{i}, \rho(t)\right\}\right],
\end{aligned}
$$

where we defined the vector of quadrature operators

$$
\hat{\mathbf{R}}=\left(\hat{Q}, \hat{P}, \hat{X}_{b}, \hat{P}_{b}\right),
$$

while $\gamma$ is the damping matrix:

$$
\gamma=\left(\begin{array}{cccc}
\frac{\kappa}{2} & -i \frac{\kappa}{2} & 0 & 0 \\
i \frac{\kappa}{2} & \frac{\kappa}{2} & 0 & 0 \\
0 & 0 & \frac{\Gamma_{m}}{2}\left(2 \bar{n}_{m}+1\right) & -i \frac{\Gamma_{m}}{2} \\
0 & 0 & i \frac{\Gamma_{m}}{2} & \frac{\Gamma_{m}}{2}\left(2 \bar{n}_{m}+1\right)
\end{array}\right),
$$

$\bar{n}_{m}=1 /\left(e^{\hbar \omega_{m} / k_{B} T}-1\right)$ is the mean occupancy of the mechanical oscillator, $k_{B}$ is the Boltzmann constant and $T$ is the temperature of the mechanical reservoir [1, 2]. We note that, in choosing a Lindblad form, we automatically excluded the use of the standard Brownian motion master equation (SBMME) 22 to describe mechanical damping. Indeed, a Lindblad form greatly simplifies our analysis, since it avoids non-positivity issues that are known to occur in the SBMME [23].

\section{A. Bi-linear approximation of the model}

The main effect of the drive is to displace the steady states of both the cavity field and the mechanical position [2. We assume that the cavity is driven sufficiently strongly (and that the optomechanical couplings are weak enough) so that the system dynamics can be approximated via a bilinear master equation description, where only small quantum fluctuations around the semi-classical steady 
state are considered [2, 17. In detail, we start by displacing our canonical operators as per $\hat{\mathbf{R}} \rightarrow \hat{\mathbf{R}}+\mathbf{R}_{0}$, where

$$
\mathbf{R}_{0}=\left(Q_{0}, P_{0}, x_{0}, p_{0}\right)
$$

is the vector of steady-state quadrature averages. Here, $x_{0}$ and $p_{0}$ are the average position and momentum of the mechanics in the steady state, while $Q_{0}$ and $P_{0}$ are the steady state displacements of the amplitude and phase quadratures, respectively [2]. Of course, the steady-state expectation values of the transformed operators will now vanish. This results in the following equations for the steady-state values of the system's first moments:

$$
\begin{aligned}
Q_{0} & =\frac{-2 \Delta_{\text {eff }} \mathcal{E}}{\sqrt{2}\left(\Delta_{\text {eff }}^{2}+\frac{\kappa^{2}}{4}\right)} \\
P_{0} & =\frac{-\kappa \mathcal{E}}{\sqrt{2}\left(\Delta_{\text {eff }}^{2}+\frac{\kappa^{2}}{4}\right)} \\
x_{0} & =-\frac{\omega^{\prime}(0) \omega_{m} \mathcal{E}^{2}}{\left(\omega_{m}^{2}+\frac{\Gamma_{m}^{2}}{4}\right)\left(\Delta_{\text {eff }}^{2}+\frac{\kappa^{2}}{4}\right)+\omega^{\prime \prime}(0) \omega_{m} \mathcal{E}^{2}} \\
p_{0} & =\frac{\Gamma_{m}}{2 \omega_{m}} x_{0}
\end{aligned}
$$

where $\Delta_{\text {eff }}=\Delta_{0}-\sqrt{2} g_{1} x_{0}+g_{2} x_{0}^{2}$ is the effective detuning. The non-linearity of Eqs. 110 13 suggests that multiple steady state solutions are possible 1, 2, This is known as dynamical multistability 1, 2]. In detail, depending on the driving strength up to five (quadratic model) or three (linear model) different steady states solutions can exist. In this work, we only focus on parameters regimes where the system is stable, i.e., where a unique real solution to Eqs. (10 13) exists. This in turn places an upper bound to the drive strength $|\mathcal{E}|^{2}[11$.

After the displacement has been implemented, we neglect terms that are beyond quadratic order in the transformed canonical operators 2. The corresponding master equation reads

$$
\begin{aligned}
\dot{\rho}(t)= & -\frac{i}{\hbar}\left[H_{B}, \rho(t)\right] \\
& +\sum_{i j} \frac{\gamma_{i j}}{2}\left[2 \hat{R}_{i} \rho(t) \hat{R}_{j}-\left\{\hat{R}_{j} \hat{R}_{i}, \rho(t)\right\}\right],
\end{aligned}
$$

where we note that the Lindblad operators remain unchanged, while the Hamiltonian now takes the bilinear form

$$
H_{B}=\frac{\hbar}{2} \Delta_{\text {eff }}\left(\hat{Q}^{2}+\hat{P}^{2}\right)+\frac{\hbar}{2} \omega_{m} \hat{P}_{b}^{2}+\frac{\hbar}{2} \omega_{\text {eff }} \hat{X}_{b}^{2}
$$

$$
+\hbar g_{e f f} \hat{X}_{b}\left(\hat{Q} Q_{0}+\hat{P} P_{0}\right)
$$

with $\omega_{\text {eff }}=\omega_{m}+2 g_{2}|\alpha|^{2}$ the effective mechanical frequency and $g_{\text {eff }}=-\sqrt{2} g_{1}+2 g_{2} x_{0}$ the effective coupling strength. Note that the assumption of strong cavity driving translates into the condition that the intracavity photon number is large, i.e., $|\alpha|^{2} \equiv\left(Q_{0}^{2}+P_{0}^{2}\right) / 2 \gg 1$.

\section{B. Limits of validity of Eq. 15).}

In the bi-linearization procedure we neglect terms that are beyond second order in the quadrature operators (nonlinear terms for brevity), under the assumption that the terms we keep are the dominant ones. We here discuss the validity of this approach and hence of Hamiltonian (15). In particular, our approach requires that the dominant nonlinear term $\propto g_{1} X_{b}\left(Q^{2}+P^{2}\right)$ is negligible compared to the bilinear terms that depend on $g_{2}$. In general, the dominant $g_{2}$-dependent bilinear term is proportional to $g_{2}|\alpha|^{2}$. Hence, a necessary condition for the validity of our approximations (within the framework of the quadratic model) is $g_{2} \gg g_{1} /|\alpha|^{2}$ - note that this is effectively a constraint on the driving strength. Regarding the remaining $g_{2}$-dependent terms, they may or may not be negligible depending on the driving strength considered. At high enough drive strengths we expect that all (bilinear) terms should be kept to maximise the accuracy of our approximate model. Conversely at lower driving powers, where only the term $\propto g_{2}|\alpha|^{2}$ should be kept, the effect of the additional $g_{2}$-dependent contributions is so minuscule that their inclusion (or otherwise) has no visible effect on the QFI. Therefore, we conclude that all bilinear terms in our Hamiltonian (15) can be kept, provided that the condition $g_{2} \gg g_{1} /|\alpha|^{2}$ is satisfied. We checked numerically that this argument indeed holds for the drive strengths considered in this manuscript.

Finally, we note that the above discussion depends on the parameters $g_{1}, g_{2}$, which are a priori unknown in our scenario of interest. Yet, it is worth pointing out that in practical situations one would not start the quantum estimation procedure with complete ignorance of the parameters: at least an orderof-magnitude estimate is typically available (e.g. via first principles modelling). The validity conditions for Eq. 15) can then be checked in the worst-case scenario compatible with the initial rough estimates for $g_{1}, g_{2}$. 


\section{ESTIMATING COUPLING CONSTANTS FROM THE STEADY STATE}

\section{A. Covariance Matrix Formalism}

Due to its bilinear form, the master equation (14) admits a Gaussian steady state that can, in general, be fully characterised by its first and second moments of the quadrature operators $\hat{\mathbf{R}}$ [14]. After having determined our steady state, we will be able to exploit general closed-form expressions that are available for QET in Gaussian models [15.

As anticipated in the previous section, the first moments of our Gaussian steady state are given by $\mathbf{R}_{0}=\left(Q_{0}, P_{0}, x_{0}, p_{0}\right)$, and are found by solving Eqs. 10 13 - recall also that we will only consider parameter regimes in which such solution is unique. The second moments are instead encoded in the steady state covariance matrix $\bar{\sigma}$, which in our displaced frame of reference is given by 14

$$
\overline{\boldsymbol{\sigma}}=\left[\begin{array}{cccc}
\left\langle\hat{Q}^{2}\right\rangle & \left\langle\frac{1}{2}\{\hat{Q}, \hat{P}\}\right\rangle & \left\langle\hat{Q} \hat{X}_{b}\right\rangle & \left\langle\hat{Q} \hat{P}_{b}\right\rangle \\
\left\langle\frac{1}{2}\{\hat{Q}, \hat{P}\}\right\rangle & \left\langle\hat{P}^{2}\right\rangle & \left\langle\hat{P} \hat{X}_{b}\right\rangle & \left\langle\hat{P} \hat{P}_{b}\right\rangle \\
\left\langle\hat{X}_{b} \hat{Q}\right\rangle & \left\langle\hat{X}_{b} \hat{P}\right\rangle & \left\langle\hat{X}_{b}^{2}\right\rangle & \left\langle\frac{1}{2}\left\{\hat{X}_{b}, \hat{P}_{b}\right\}\right\rangle \\
\left\langle\hat{P}_{b} \hat{Q}\right\rangle & \left\langle\hat{P}_{b} \hat{P}\right\rangle & \left\langle\frac{1}{2}\left\{\hat{X}_{b}, \hat{P}_{b}\right\}\right\rangle & \left\langle\hat{P}_{b}^{2}\right\rangle
\end{array}\right],
$$

where $\{\hat{A}, \hat{B}\} \equiv \hat{A} \hat{B}+\hat{B} \hat{A}$ is the anticommutator. As detailed in Appendix A master equation (14) implies the following Lyapunov equation for the steady state covariance matrix:

$$
B^{T} \overline{\boldsymbol{\sigma}}+\overline{\boldsymbol{\sigma}} B=C
$$

where

$$
\begin{aligned}
B & =\frac{i}{\hbar} \mathbf{H W}+\gamma_{\mathbf{A}} \mathbf{W}, \\
C & =-\mathbf{W} \gamma_{\mathbf{s}} \mathbf{W}, \\
\mathbf{H} & =\left(\begin{array}{cccc}
\hbar \Delta_{\text {eff }} & 0 & \hbar g_{\text {eff }} Q_{0} & 0 \\
0 & \hbar \Delta_{\text {eff }} & \hbar g_{\text {eff }} P_{0} & 0 \\
\hbar g_{\text {eff }} Q_{0} & \hbar g_{\text {eff }} P_{0} & \hbar \omega_{\text {eff }} & 0 \\
0 & 0 & 0 & \hbar \omega_{m}
\end{array}\right), \\
\mathbf{W} & =\left(\begin{array}{cccc}
0 & i & 0 & 0 \\
-i & 0 & 0 & 0 \\
0 & 0 & 0 & i \\
0 & 0 & -i & 0
\end{array}\right), \\
\gamma_{\mathbf{S}} & =\frac{\gamma+\gamma^{T}}{2}, \\
\gamma_{\mathbf{A}} & =\frac{\gamma-\gamma^{T}}{2} .
\end{aligned}
$$

We note that Eq. 17 can be solved analytically in terms of the model parameters and the vector of averages $\mathbf{R}_{0}$. The latter, however, may in general not admit an analytical expression in terms of the model parameters, as we recall it is the solution to the nonlinear system of equations $\sqrt{10}-(13)$. In the next section we shall show how to develop a comprehensive QET analysis of the coupling parameters solely from the knowledge of the first and second moments of our Gaussian steady state.

\section{B. Quantum Estimation Theory for Gaussian States}

The aim of quantum estimation theory (QET) is to identify the best strategy for estimating one or more parameters encoded in the density matrix of a quantum system 911. Here we focus on local QET, which seeks a strategy that maximises the Fisher information over all possible measurements, and implicitly assumes that a rough estimate of the parameter value is known in advance [9].

In our model of driven-dissipative optomechanics, the parameters to be estimated shall be the coupling strengths $g_{1}$ and $g_{2}$. As anticipated, all of the information about these parameters will be contained in the steady-state averages, $\mathbf{R}_{0}$, as well as in the steady state covariance matrix, $\overline{\boldsymbol{\sigma}}$. Specifically, for our coupling parameters $\left(g_{1}, g_{2}\right)$ the elements of the quantum Fisher information matrix (QFIM) are given by

$$
\begin{aligned}
l_{i, j} & =\left(\partial_{g_{i}} \mathbf{R}_{0}^{T}\right) \overline{\boldsymbol{\sigma}}^{-1}\left(\partial_{g_{j}} \mathbf{R}_{0}\right) \\
& +2 \operatorname{Tr}\left[\left(\partial_{g_{i}} \overline{\boldsymbol{\sigma}}\right)\left(4 \mathcal{L}_{\overline{\boldsymbol{\sigma}}}+\mathcal{L}_{W}\right)^{-1}\left(\partial_{g_{j}} \overline{\boldsymbol{\sigma}}\right)\right],
\end{aligned}
$$

where $\mathcal{L}_{\overline{\boldsymbol{\sigma}}}(\mathbf{A})=\overline{\boldsymbol{\sigma}} \mathbf{A} \overline{\boldsymbol{\sigma}}, \mathcal{L}_{W}(\mathbf{A})=$ WAW. $\quad$ Note also that the term $\left(4 \mathcal{L}_{\bar{\sigma}}+\mathcal{L}_{W}\right)^{-1}$ refers to the pseudoinverse if the term inside the bracket is singular 15, 24. The first term is the contribution due to the averages, while the second term is the contribution due to the variances and covariances towards each QFI matrix element 24]. This terminology will be convenient later on as we seek to unravel how the different terms contribute across different parameter regimes. We note, however, that our terminology only describes the origin of the dependence of the gradients with respect to the coupling parameters. Hence whilst the first term in eq. 24 only contains gradients of the averages with respect to the coupling constants, and we will therefore call it the contribution of the averages, it does also depend on the covariance matrix. Eq. (24) facilitates efficient numerical computation of the QFI [24].

The ultimate limit to parameter estimation in this context is set by the QCRB [9, 12, 13. In multiparameter estimation theory both coupling param- 
eters are assumed to be unknown (or only known with low precision). In this case, the QCRB relates the covariance matrix of any pair of unbiased estimators for the parameters $\left(g_{1}, g_{2}\right)$ to the QFIM. For $M$ experimental runs, the corresponding QCRB reads [13, 15

$$
\operatorname{Cov}\left(g_{1}, g_{2}\right) \geq \frac{1}{M} \mathbf{I}^{-1}
$$

The limiting case of single-parameter estimation theory can be reached if we assume that only one parameter is unknown, say $g_{i}$. In this case the QCRB relates the variance $\operatorname{Var}\left(g_{i}\right)$ of an unbiased estimator of the parameter $g_{i}$ to the corresponding diagonal element of the QFIM. For $M$ experimental runs, the corresponding bound reads [15, 16, 24, 27.

$$
\operatorname{Var}\left(g_{i}\right) \geq \frac{1}{M I_{i i}}
$$

In other words, the diagonal elements of the QFI matrix quantify the "best-case-scenario" performance for the estimation of each individual parameter. Hence, in what follows we shall pick Eq. (26) as our benchmark in evaluating the performance of various measurements (see below). For brevity, we shall also refer to $l_{11}$ as the " $g_{1}-\mathrm{QFI",} \mathrm{and} l_{22}$ as the " $g_{2}$-QFI". Note that in single-parameter estimation theory the saturation of the QCRB is guaranteed, at least in the limit $M \rightarrow \infty$, and assuming that every mathematically allowed quantum measurement can be implemented [13, 15, 24]. This, however, is not true for the estimation of multiple parameters: in this case the optimal measurements for different parameters may not be compatible [13].

While the QFI quantifies the ultimate quantum limit to parameter estimation [9, 13, 28, the estimation performance of specific measurement strategies may be quantified via the classical Fisher information (FI) matrix [9, 29]. In our context, the FI measures the amount of information that a classical random variable $s$ (the outcome of a quantum measurement) contains about the parameters $g_{1}, g_{2}$ 30. The FI matrix elements take the form

$$
J_{i, j}=\int_{-\infty}^{\infty} d s \frac{1}{p_{g_{1}, g_{2}}(s)}\left(\frac{\partial p_{g_{1}, g_{2}}(s)}{\partial g_{i}}\right)\left(\frac{\partial p_{g_{1}, g_{2}}(s)}{\partial g_{j}}\right),
$$

where $p_{g_{1}, g_{2}}(s)$ is the probability distribution of the measurement outcome $s$, assumed to be a smooth function of $\left(g_{1}, g_{2}\right)$ [15, 30]. Depending on the chosen observables, analytical solutions to the integral in Eq. 27 may exist. This is particularly true for quadrature measurements (i.e. a measurement of $\hat{Q}, \hat{P}, \hat{X}_{b}, \hat{P}_{b}$ or a linear combination thereof), provided that the measured state is Gaussian [16].

In the case of optomechanics, it is well known that one can use a homodyne detection scheme to measure the light quadratures $\hat{Q}, \hat{P}[16,31$. However, we shall also consider a direct measurement of the mechanical quadratures, $\hat{X}_{b}$ and $\hat{P}_{b}$, for completeness. In practice this could potentially be achieved using e.g. another optical mode of the cavity. In this scenario, the probability distribution associated with a measurement of $\hat{s} \in\left\{\hat{Q}, \hat{P}, \hat{X}_{b}, \hat{P}_{b}\right\}$ has the following expression [16]:

$$
p_{g_{1}, g_{2}}(s)=\frac{e^{-\frac{\left(s-s_{0}\left(g_{1}, g_{2}\right)\right)^{2}}{2 \bar{\sigma}_{k k}\left(g_{1}, g_{2}\right)}}}{\sqrt{2 \pi \bar{\sigma}_{k k}\left(g_{1}, g_{2}\right)}}
$$

where $s_{0}\left(g_{1}, g_{2}\right)$ is the steady state average of the chosen quadrature, appropriately chosen from the set $\left\{Q_{0}, P_{0}, x_{0}, p_{0}\right\}$, while $\bar{\sigma}_{k k}\left(g_{1}, g_{2}\right)$ is the corresponding diagonal element of the steady state covariance matrix $\left(\bar{\sigma}_{11}\right.$ for $\hat{s}=\hat{Q}, \bar{\sigma}_{22}$ for $\hat{s}=\hat{P}$ and so on). In this setting an analytical solution to the integral in Eq. 27) exists and is given by

$$
\begin{aligned}
J_{i, j} & =\frac{1}{2 \bar{\sigma}_{k k}\left(g_{1}, g_{2}\right)^{2}} \times \\
& {\left[2 \bar{\sigma}_{k k}\left(g_{1}, g_{2}\right)\left(\frac{\partial s_{0}\left(g_{1}, g_{2}\right)}{\partial g_{j}}\right)\left(\frac{\partial s_{0}\left(g_{1}, g_{2}\right)}{\partial g_{i}}\right)\right.} \\
& \left.+\left(\frac{\partial \bar{\sigma}_{k k}\left(g_{1}, g_{2}\right)}{\partial g_{j}}\right)\left(\frac{\partial \bar{\sigma}_{k k}\left(g_{1}, g_{2}\right)}{\partial g_{i}}\right)\right] .
\end{aligned}
$$

Note that the choice of a strategy to estimate the parameters $\left(g_{1}, g_{2}\right)$ is optimal if the FI and QFI matrices are equal, i.e. $\mathbf{J}=\mathbf{I}$.

As anticipated, we shall focus solely on the diagonal elements of the QFIM (Eq. (24)): $I_{1,1}$ and $I_{2,2}$, respectively. As noted above, the diagonal elements are indeed the "most optimistic" quantifiers of estimation precision of the coupling strengths. In general, however, the combined precision of the two parameter estimations will be worse than what the diagonal elements suggest.

Both, the definitions of the QFI (Eq. (24)) and the FI (Eq. (29) ) rely on the derivatives of the steady state covariance matrix and the averages with respect to the coupling strengths. Since we have seen that both $\bar{\sigma}$ and $\mathbf{R}_{0}$ are determined by the nonlinear system of equations (10)- 13 , which in general can only be solved numerically, we use implicit differentiation to calculate the derivatives in question. This allows us to express all our quantities of interest in 
terms of the numerical solution to the above nonlinear equations, and allows us to avoid numerical differentiation altogether.

\section{RESULTS}

In this section we explore how the achievable estimation precision of the coupling strengths is impacted by temperature and drive amplitude, while also highlighting similarities and differences between the quadratic and linear models. For concreteness, we choose parameters matching those used in a recent experiment employing a microwave cavity, where the ground state of a mechanical oscillator was approached via back-action cooling arising from a red-detuned laser drive 33. Correspondingly, we adopt the following parameter values $\omega_{m}=1.1 \times 10^{7}$ $\mathrm{Hz}, m=4.8 \times 10^{-14} \mathrm{~kg}, \Gamma_{m}=32 \mathrm{~Hz}, \Delta_{0}=\omega_{m}$, $\kappa=10^{5} \mathrm{~Hz}$ and $g_{1}=2 \times 10^{2} \mathrm{~Hz} 33$. In order to ensure that the driving is strong enough for the Gaussian approximation to hold, and in order to avoid stability issues in the linear model, we consider a range of driving amplitudes $10^{8} \leq \mathcal{E} \leq 3.8 \times 10^{9} \mathrm{~Hz}$. In terms of the intracavity photon number, $|\alpha|^{2}$, this corresponds to the range $80 \lesssim|\alpha|^{2} \lesssim 1.2 \times 10^{5}$ [or $\left.1.9 \lesssim \log _{10}\left(|\alpha|^{2}\right) \lesssim 5.1\right]$. For the quadratic model we additionally need to ensure that the Hamiltonian (15) is valid, for which we require $g_{2} \gg g_{1} /|\alpha|^{2}$ - see Section II B. We therefore choose a value of $g_{2}=10$ $\mathrm{Hz}$ to ensure the latter condition is satisfied across the whole range of intracavity photon numbers considered. Although such values of the quadratic coupling strength are not compatible with the set-up in 33, they can be achieved in others. One such set-up is featured in [34, where the authors consider $g_{2}=0.07 g_{1}$, which is the same order of magnitude as in our work. As an interesting aside, a membrane-in-the-middle optomechanical system allows for great flexibility in the choice of both $g_{1}$ and $g_{2}$, so that both regimes $g_{1}>g_{2}$ and $g_{2}<g_{1}$ are in principle possible [2, 32].

Notice also that we have chosen to work in the red-detuned regime. Our choice is motivated by two factors. Firstly, red-detuning gives rise to backaction cooling whereby the second moments of the mechanical quadratures can become strongly dependent on the drive and coupling strengths (particularly at finite temperatures). As we shall see, this leads to interesting behavior in the QFI. Secondly, the back-action in this regime enhances the mechanical damping avoiding the dynamical instabilities that arise in the blue-detuned regime, thus ensuring that the Gaussian formalism remains applicable.
In future work, our approach will be applicable to other choices of the detuning provided that the stability conditions discussed in Appendix $B$ are met. The results will in general depend on the specific detuning value chosen.

We examine three scenarios: zero temperature $(T=0 \mathrm{~K})$, low temperature $(T=1 \mathrm{mK})$ and "high" temperature $(T=80 \mathrm{mK})$. In each case we are looking for the best strategy to estimate the linear, $g_{1}$, and quadratic, $g_{2}$, coupling strengths. First, we establish the fundamental quantum limits on the estimation precision, which, in accordance with the quantum Cramér-Rao bound (QCRB), are quantified with the "global" QFIs (i.e. the QFIs calculated from the bipartite state of light plus mechanics). Additionally, by tracing out the mechanical (light) mode, we can calculate "local" QFIs that are relevant when only the light (mechanical) mode is directly measurable. Comparing these local QFIs with the global ones will also reveal how much information about the coupling parameters is contained in the reduced states of light and mechanics. Finally, we compare the QFI limits to the performance of a small selection of "realistic" measurements (quantified with the respective FI), including those of $\hat{Q}, \hat{P}$, $\hat{X}_{b}$ and $\hat{P}_{b}$. This can help us discern which of the experimentally common measurements constitute the best strategy to parameter estimation in each scenario. In most of our reported results we have chosen to measure the estimation precision with the "single shot" relative error, which obeys the inequality

$$
\frac{\Delta g_{i}}{g_{i}} \geq \frac{1}{g_{i} \sqrt{l_{i, i}}} \equiv \delta g_{i}^{\min }
$$

Note how Eq. 30 is a consequence of the QCRB for the case of $M=1$ experimental runs [the case of $M>1$ experimental runs can be trivially obtained by dividing the right hand side of Eq. 30 by $\sqrt{M}$.

In Fig. 2(a) we investigate the effects of the higher order $g_{2}$ term, temperature and driving on the estimation precision of the linear coupling strength, $g_{1}$. Overall, $\delta g_{1}^{\min }$ displays a surprisingly complex dependence on temperature. There is a crossover around $\log _{10}\left(|\alpha|^{2}\right) \sim 4.7$ (or $|\alpha|^{2} \sim 5 \times 10^{4}$ ): below this value the high temperature scenario offers the best precision for estimating $g_{1}$, but above it the best precision is found at lower temperatures. Additionally, pronounced local maxima in $\delta g_{1}^{\min }$ are observed for the linear model at nonzero temperature, as well as the quadratic model in the low temperature scenario. In contrast, $\delta g_{1}^{\min }$ shows a comparatively weak dependence on $|\alpha|^{2}$ for the quadratic model at high temperature. 

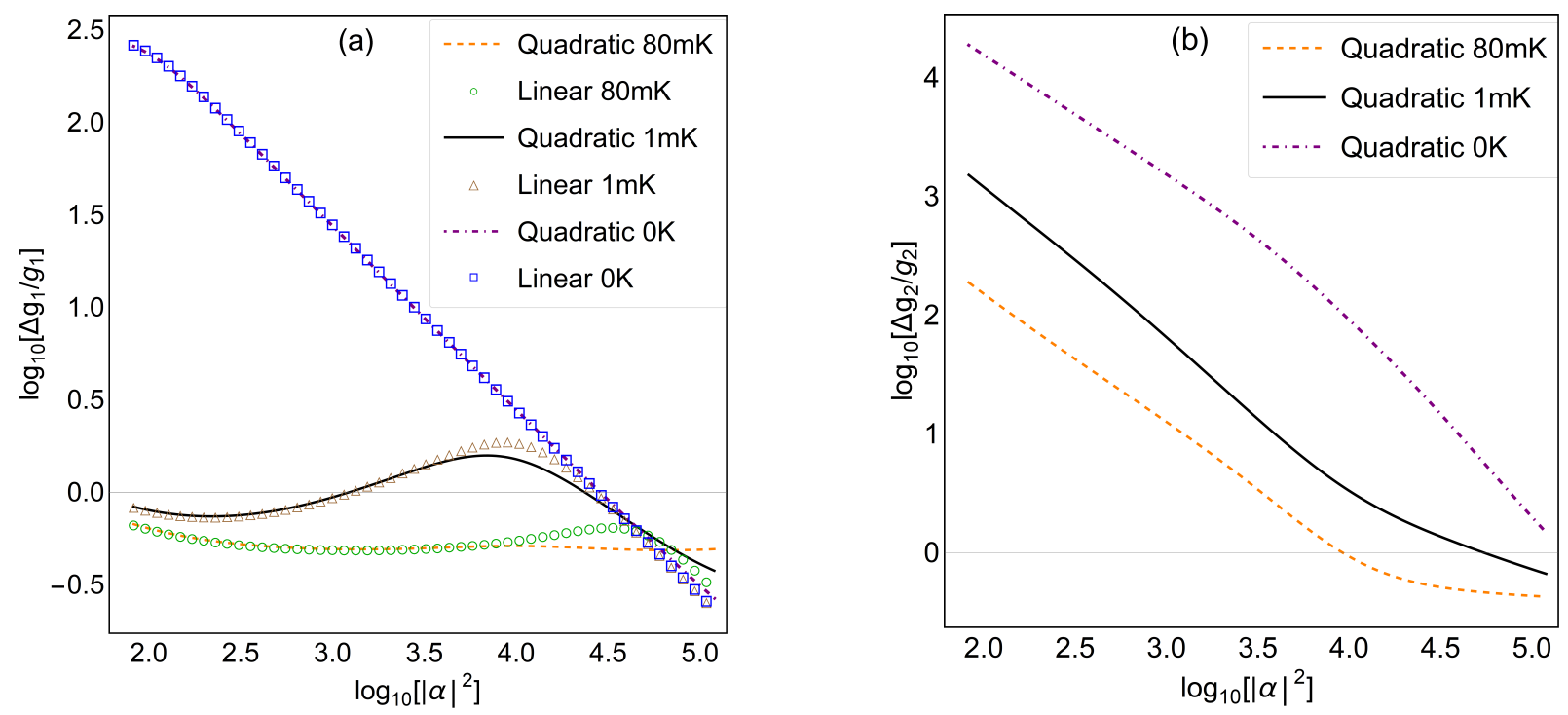

FIG. 2: (a) Log-log plot of the relative error bound on $g_{1}, \delta g_{1}^{\min }$, against the intracavity photon number $|\alpha|^{2}$. The plot compares linear and quadratic models in the zero temperature (blue squares and purple dot-dashed line, respectively), low temperature (brown triangles and black line, respectively) and high temperature (green circles and orange dashed line, respectively) scenarios. (b) Log-log plot of the relative error bound on $g_{2}, \delta g_{2}^{\min }$, against the intracavity photon number, $|\alpha|^{2}$ as predicted by the quadratic model in the zero temperature (purple dot-dashed line), low temperature (black line) and high temperature (orange dashed line) scenarios.

As discussed in Appendix B (see in particular Fig. 5), this behavior can be partly understood by looking at the relative contributions of the variances and averages to the QFI [see discussion below Eq. [24]], and noting how these contributions change with temperature. The contribution of the averages to the $g_{1}$-QFI increases monotonically with the intracavity photon number, and eventually provides the dominant contribution to the $g_{1}-\mathrm{QFI}$ in all the explored examples - with the exception the high temperature quadratic model, as explained below. However, for non-zero temperatures the contribution of the variances to the $g_{1}-$ QFI is important, and it is in fact dominant at sufficiently low driving. In the region of driving strengths where linear and quadratic models are in agreement, the important role played by the contribution of the variances can be related to the cavity-assisted cooling of the mechanics 1, 2, which makes the effective mechanical temperature (hence the system's covariance matrix) strongly coupling-dependent. Note also that the impact of the cooling effect gets stronger at higher temperatures. The local maximas observed in $\delta g_{1}^{\mathrm{min}}$ at finite temperature occur close to the crossover point between the contributions of the averages and variances. In contrast, at zero temperature the contri- bution of the variances is very weakly dependent on $g_{1}$, so that in that case the averages always dominate and no local maximum is observed. Additionally, the fact that the contribution of the averages to the $g_{1}-$ QFI involves the inverse of the covariance matrix [see Eq. (24)] suggests that the best precision may eventually be expected when the mechanical bath is at zero-temperature. As anticipated, the quadratic model at $80 \mathrm{mK}$ provides an exception to the above discussion: there $\delta g_{1}^{\min }$ is dominated by the (approximately constant) contribution from the variances to the $g_{1}-$ QFI for all the considered driving strengths.

In Fig. 2(b) we explore the effect of cavity driving and temperature on the estimation precision of the quadratic coupling strength, $g_{2}$. At low intracavity photon numbers the relative error bounds are significantly larger for $g_{2}$ than $g_{1}$. At high intracavity photon numbers $\left(\log _{10}|\alpha|^{2} \gtrsim 5\right)$, however, $\delta g_{1}^{\min }$ and $\delta g_{2}^{\min }$ become comparable, reflecting the fact that $g_{2}$ is amplified by a factor $|\alpha|^{2}$ in Hamiltonian (15).

Interestingly, for all $|\alpha|$ within our allowed range the high temperature scenario predicts the lowest relative errors bound on $g_{2}$ : a hotter mechanical bath gives a (potentially) better estimation precision for all driving strengths studied. This can be 

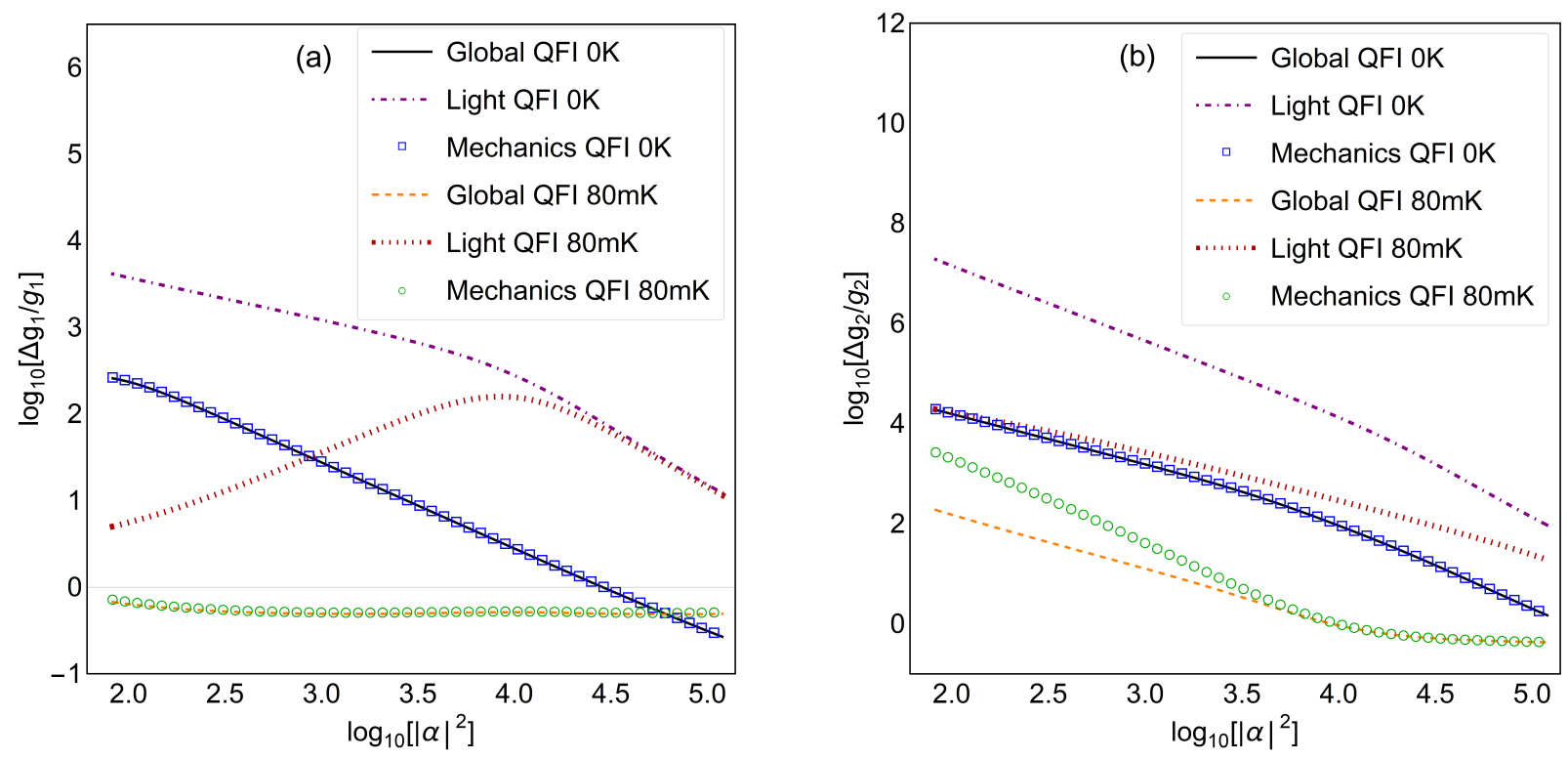

FIG. 3: Relative error bounds on coupling constants (a) $g_{1}$ and (b) $g_{2}$, as a function of the intracavity photon number, $|\alpha|^{2}$ as implied by the global, light and mechanics QFIs in the case of zero temperature (black line, orange dashed line, blue squares, respectively) and high temperature (purple dot-dashed line, red dotted line and green circles, respectively).

traced back to the fact that, also in the estimation of $g_{2}$, the information content of the variances increases with temperature. In this case the contribution of the variances is always higher than that of the averages at lower driving strengths and for sufficiently high temperatures it remains so over the full range of driving strengths - see Appendix. B. The overall result is that the relative error bounds on $g_{2}$ decrease monotonically with increasing drive.

In Fig. 3 we compare global and local QFIs for $g_{1}$ (Fig. 3(a)) and $g_{2}$ (Fig. 3(b)). In a nutshell, we find that the majority of information about the coupling parameters is contained in the reduced state of the mechanics. Note that, in standard optomechanical experiments, measurements are typically performed on the light mode. Nevertheless, our results suggest that significantly more information about the couplings might be available by probing the mechanical motion more directly.

For $g_{1}$ the precision bounds obtained from either the mechanical subsystem, or the optical subsystem, drop monotonically with drive strength at $T=0$, but display more complex behavior at nonzero temperature. This matches what happens with the precision bounds of the full system. Indeed, the $g_{1}$ QFI of the mechanical subsystem is very close to the global $g_{1}$-QFI for all the considered temperatures. However, while the $g_{2}-\mathrm{QFI}$ of the mechani- cal subsystem is close to the global one for $T=0$, for $T=80 \mathrm{mK}$ there is a visible gap between the two that only closes at strong enough driving, i.e. $\log _{10}\left(|\alpha|^{2}\right) \gtrsim 3.8$.

Finally, in Fig. 4 we show how some realistic measurements perform in comparison to the ultimate limits given by the $g_{1}-$ and $g_{2}-$ QFIs. Out of the measurements considered, the mechanical position almost always does best at estimating the coupling parameters. The ultimate limits to the estimation precision of the coupling strengths can only be approached at zero temperature, usually by measurement of $\hat{X}_{b}$. For higher temperatures this limit is never achieved for either coupling parameter. Notice also the unusual behaviour of the measurement of $P$ in the case of $g_{2}$ at $T=80 \mathrm{mK}$. The two peaks correspond to two minima of the classical FI, where the information about $g_{2}$ is very close to zero.

\section{CONCLUSIONS}

We employed local QET to the problem of estimating linear and quadratic coupling parameters in driven-dissipative optomechanics. For experimentally realistic values of the model parameters, inspired by Refs. 33 and 34, we found that at low intracavity photon numbers the linear coupling 

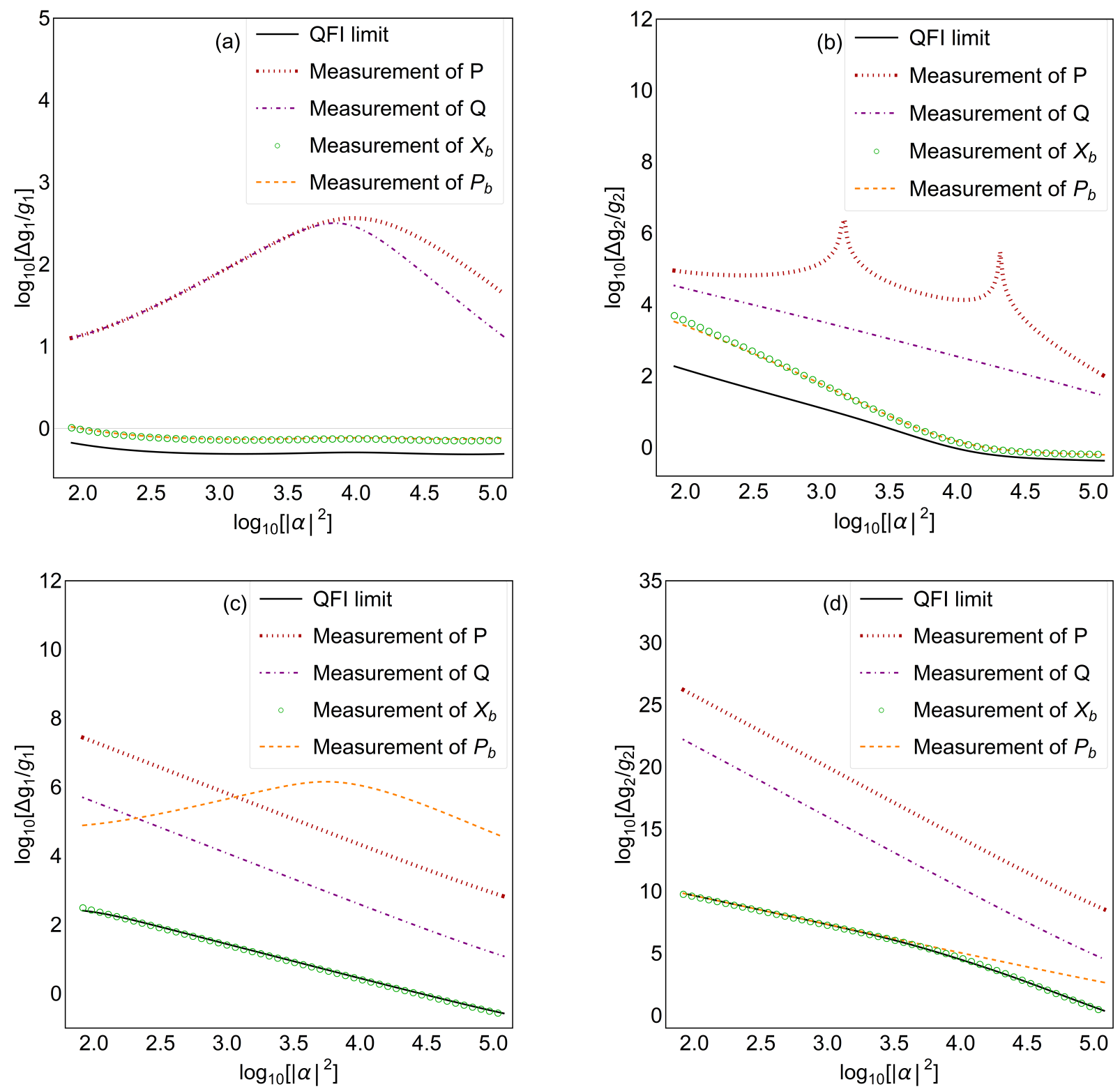

FIG. 4: Log-log plots of relative error bounds for coupling constants against the intracavity photon number, $|\alpha|^{2}$ as implied by the QFI (black line) and the measurements of $\hat{P}$ (red dotted line), $\hat{Q}$ (purple dot-dashed line), $\hat{X}_{b}$ (green circles) and $\hat{P}_{b}$ (orange dashed line). (a) and (b) are for $g_{1}$ and $g_{2}$ in the high temperature scenario respectively, whilst (c) and (d) are for $g_{1}$ and $g_{2}$ in the zero-temperature scenario.

strength is easier to estimate than the quadratic one. At high intracavity photon numbers, however, their estimation precisions become comparable. Our analysis also shows that, at zero temperature, a measurement of the mechanical quadrature $\hat{X}_{b}$ is the best possible choice to a very good approximation.

Exploring the effect of temperature on the esti- mation precision of the coupling strengths, we found that higher temperatures are not always detrimental to the estimation performance. Temperature has a particularly striking effect in the estimation of the quadratic coupling parameter: in this case we found that a hotter mechanical bath resulted in a higher estimation precision for all drive strengths consid- 
ered. In contrast, in the case of the linear coupling strength the effect of temperature is most significant at lower driving. Past a certain drive strength, better estimation precision for the linear coupling parameter is achieved at lower temperatures.

In future work, one could investigate how the estimation precision of $g_{1}, g_{2}$ may be affected by the inclusion of anharmonicities in the mechanical potential [37], and by different choices of detuning. Additionally, our methods could be extended to purely quadratic settings $\left(g_{1}=0\right)$, or to cases where $g_{2}>g_{1}$.

\section{ACKNOWLEDGMENTS}

We thank M. G. Genoni for the enlightening discussions about the validity of the "quadratic model" employed in this manuscript. T.T. acknowledges support from the University of Nottingham via a Nottingham Research Fellowship. K.S. and A.D. acknowledge support from the University of Nottingham. A.D.A. was supported through a Leverhulme Trust Research Project Grant (RPG-2018-213).

\section{Appendix A: Covariance matrix language}

In this section we introduce the covariance matrix language. This is particularly convenient for Gaussian states, which can be fully characterised by their first and second moments [16].

Let us consider a system of $N$ bosonic modes, described by a vector of quadratures $\hat{\mathbf{R}}=$ $\left(\hat{X}_{1}, \hat{P}_{1}, \ldots, \hat{X}_{N}, \hat{P}_{N}\right)$ [14]. The commutator between any two quadrature operators is given by the corresponding element of a matrix of commutators $\mathbf{W}$ which, by construction, satisfies $\mathbf{W}^{\boldsymbol{\top}}=-\mathbf{W}$. Symbolically, the elements of $\mathbf{W}$ are

$$
W_{i j}=\left[\hat{R}_{i}, \hat{R}_{j}\right] \text {. }
$$

In the main text we are dealing with open system dynamics which can be approximated via a bilinear master equation of the general form

$$
\begin{aligned}
\dot{\rho}(t) & =-\frac{i}{\hbar}\left[H^{\prime}, \rho(t)\right] \\
& +\sum_{i j} \frac{\gamma_{i j}}{2}\left[2 \hat{R}_{i} \rho(t) \hat{R}_{j}-\left\{\hat{R}_{j} \hat{R}_{i}, \rho(t)\right\}\right],
\end{aligned}
$$

where

$$
H^{\prime}=\frac{1}{2} \sum_{i j} H_{i j} \hat{R}_{i} \hat{R}_{j}=\frac{1}{2} \hat{\mathbf{R}}^{T} \mathbf{H} \hat{\mathbf{R}}
$$

is the bilinear Hamiltonian, $\mathbf{H}$ is the Hamiltonian matrix and $\gamma_{i j}$ is the corresponding damping rate [16. Eq. A3 is the general expression for a strictly quadratic Hamiltonian. Without loss of generality we may assume that $\mathbf{H}$ is a symmetric matrix, that is $\mathbf{H}=\mathbf{H}^{T}$. If the master equation admits a steady state, the latter will be Gaussian provided that the additional condition $\mathbf{H}>0$ is satisfied [14, 16]. The first moments $\mathbf{R}_{0}$ of a quantum state $\rho(t)$ form a vector of average values defined as 35,36 .

$$
\mathbf{R}_{0}=\langle\hat{\mathbf{R}}\rangle=\operatorname{Tr}[\rho(t) \hat{\mathbf{R}}],
$$

while the second moments $\boldsymbol{\sigma}$ are encoded in the covariance matrix with elements [14, 16, 35.

$$
\sigma_{k l}=\frac{1}{2}\left\langle\left\{\hat{R}_{k}, \hat{R}_{l}\right\}\right\rangle-\left\langle\hat{R}_{k}\right\rangle\left\langle\hat{R}_{l}\right\rangle
$$

Moreover, given a master equation of the form A2 the equation of motion for the average value of a generic observable $\hat{A}$ can be deduced

$$
\frac{d}{d t}\langle\hat{A}\rangle \equiv \operatorname{Tr}[\dot{\rho}(t) \hat{A}]=\frac{i}{\hbar}\left\langle\left[H^{\prime}, \hat{A}\right]\right\rangle+\left\langle\mathcal{D}^{\dagger}(\hat{A})\right\rangle
$$

where

$$
\mathcal{D}^{\dagger}(\hat{A})=\sum_{i j} \frac{\gamma_{i j}}{2}\left(\hat{R}_{j}\left[\hat{A}, \hat{R}_{i}\right]+\left[\hat{R}_{j}, \hat{A}\right] \hat{R}_{i}\right)
$$

is the dissipator [22]. Using the same convention, we find that the vector of first moments evolves according to [14, 16]:

$$
\dot{\mathbf{R}}_{0}=-\frac{i}{\hbar} \mathbf{W} \mathbf{H R}_{0}+\mathbf{W} \gamma_{\mathbf{A}} \mathbf{R}_{0}
$$

where $\gamma$ is a matrix with elements $\gamma_{i j}$ that has been conveniently decomposed into its symmetric and antisymmetric parts:

$$
\begin{aligned}
\gamma_{\mathbf{S}} & =\frac{\gamma+\gamma^{T}}{2} \\
\gamma_{\mathbf{A}} & =\frac{\gamma-\gamma^{T}}{2}
\end{aligned}
$$

Instead, the covariance matrix obeys the following equation of motion

$$
\begin{aligned}
\dot{\boldsymbol{\sigma}} & =\left(-\frac{i}{\hbar} \mathbf{W} \mathbf{H}+\mathbf{W} \gamma_{\mathbf{A}}\right) \boldsymbol{\sigma}+\boldsymbol{\sigma}\left(\frac{i}{\hbar} \mathbf{H} \mathbf{W}+\gamma_{\mathbf{A}} \mathbf{W}\right) \\
& +\mathbf{W} \gamma_{\mathbf{S}} \mathbf{W} .
\end{aligned}
$$

The steady state covariance matrix, $\overline{\boldsymbol{\sigma}}$, can thus be found by solving the Lyapunov equation

$$
B^{T} \overline{\boldsymbol{\sigma}}+\overline{\boldsymbol{\sigma}} B=C
$$



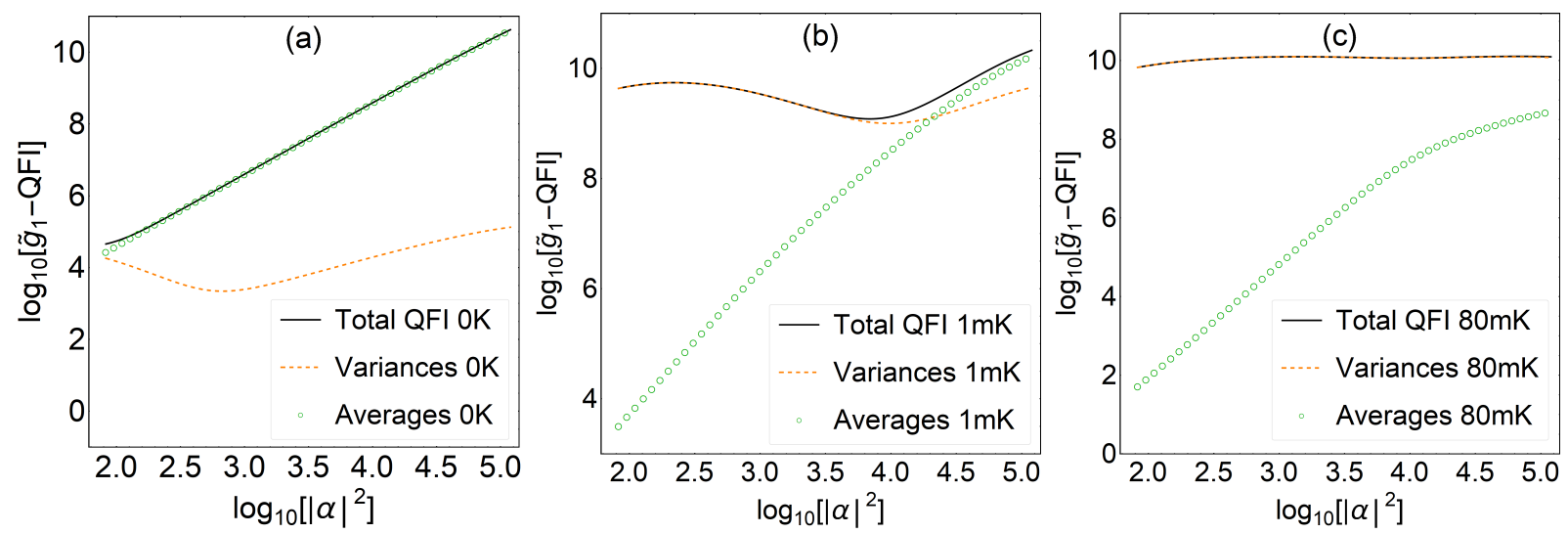

FIG. 5: Log-log plots comparing the contributions from the variances (orange dashed line) and the averages (green circles) to the $g_{1}-$ QFI in the quadratic model (black line) for $g_{1}$ in (a) the zero temperature scenario, (b) $T=1 \mathrm{mK}$, (c) $T=80 \mathrm{mK}$. The corresponding results for the linear model are not shown, but are qualitatively similar to figure (a) for zero temperature, and to (b) for both $T=1 \mathrm{mK}$ and $T=80 \mathrm{mK}$ cases.
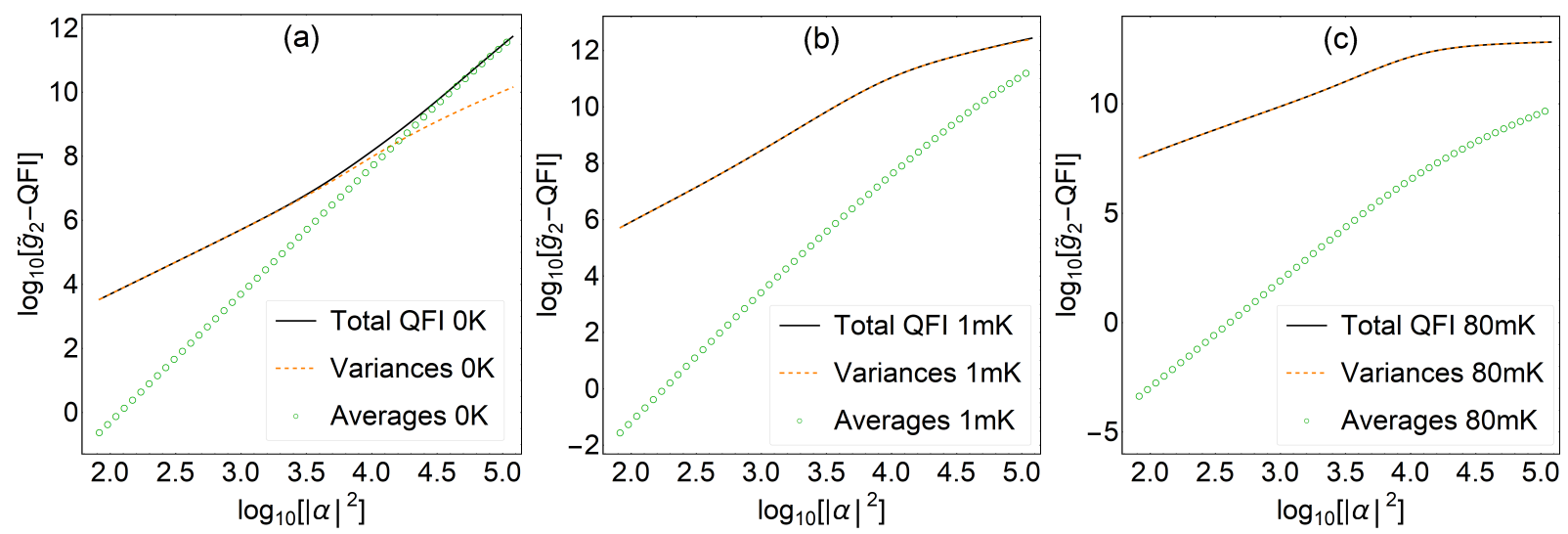

FIG. 6: Log-log plots comparing the variances (orange dashed line) and averages (green circles) contributions to QFI (black line) for $\tilde{g}_{2}$ at (a) zero temperature (b) $T=1 \mathrm{mK}$ and (c) $T=80 \mathrm{mK}$.

where

$$
\begin{aligned}
B & =\frac{i}{\hbar} \mathbf{H W}+\gamma_{\mathbf{A}} \mathbf{W}, \\
C & =-\mathbf{W} \gamma_{\mathbf{S}} \mathbf{W} .
\end{aligned}
$$

In order for the steady state covariance matrix to describe a physical state $\rho(t)$ it must be a real, symmetric and a positive semi-definite matrix [14, 16]. The semi-positivity requirement is satisfied provided that $\mathbf{B}$ is stable, i.e. the real parts of the eigenvalues of $\mathbf{B}$ are all negative [14. Additionally, the Robertson-Schrödinger uncertainty relation must hold:

$$
2 \overline{\boldsymbol{\sigma}}+\mathbf{W} \geq 0,
$$

The inequality $\mathrm{A} 15$ is in fact a necessary and sufficient condition for $\overline{\boldsymbol{\sigma}}$ to represent the steady state covariance matrix of a Gaussian state 14, 16. In our case, assuming that $\mathbf{B}$ is indeed stable, Eq. A15) can be further simplified to $\mathbf{W} \boldsymbol{\gamma}^{T} \mathbf{W} \geq 0$, which is equivalent to the simpler condition $\gamma \geq 0$. It is easy to check that the latter is always satisfied for the choice of $\gamma$ adopted in the main text.

\section{Appendix B: Non-monotonic behavior of the QFI}

In this Appendix we explore the origins of the non-monotonic behavior of the QFI when mechanical temperature is introduced, by investigating how the different contributions to the QFI (those due to the averages and the variances) behave in the zero, low and high temperature cases. 
In our set-up, the coupling strengths have dimensions of frequency, whilst the averages and the steady state covariance matrix are dimensionless. To obtain dimensionless QFIs we shall consider the dimensionless linear and quadratic coupling strengths defined as $\tilde{g}_{1}=g_{1} / \omega_{m}$ and $\tilde{g}_{2}=g_{2} / \omega_{m}$, respectively. It is straightforward to show that the QFIs for the dimensionless and the original coupling strengths are related via $\tilde{I}_{i i}=\omega_{m}^{2} I_{i i}$ for $i=\{1,2\}$.

We can now compare the contributions from the variances and averages (as defined below Eq. (24)) to the QFI in the cases of $\tilde{g}_{1}$ and $\tilde{g}_{2}$, separately.

Figure 5 shows the contributions to the $\tilde{g}_{1}-\mathrm{QFI}$ versus the steady-state intracavity photon number. In the zero temperature limit (Fig. 5(a)) the contribution of the averages dominates at all $|\alpha|$. Thus, here the majority of information about $g_{1}$ is always encoded in the averages. In contrast, in the low temperature scenario (Fig. 5(b)) we observe a crossover of the contributions from the variances and the aver- ages at $\log _{10}\left(|\alpha|^{2}\right) \sim 4$.3. In this case, the variances encode most of the $\tilde{g}_{1}-\mathrm{QFI}$ at lower drive strengths, but the contribution of the averages grows monotonically until it eventually becomes dominant. The contribution of the variances remains approximately constant with drive before declining and then eventually growing again, but at a slower rate than the contribution of the averages. For $T=80 \mathrm{mK}$ (Fig. 5(c)), we instead find that the variances contribution is always dominant (and approximately constant) for all drives considered.

In Fig. 6] we investigate the QFI contributions for $\tilde{g}_{2}$. In contrast to the $\tilde{g}_{1}$ case, the $\tilde{g}_{2}-\mathrm{QFI}$ displays monotonic bahavior as a function of the intracavity photon number. A crossover between the two contributions is only observed in the zero temperature scenario $\left(\right.$ at $\log _{10}\left(|\alpha|^{2}\right) \sim 4.2$ ), with the majority of information about $g_{2}$ contained in the variances over the whole range of $|\alpha|$ studied for $T=1 \mathrm{mK}$ and $80 \mathrm{mK}$.
[1] M. Aspelmeyer, T. Kippenberg, and F. Marquardt, Rev. Mod. Phys. 86, 1391 (2014).

[2] W. P. Bowen, and G. J. Milburn, Quantum optomechanics (CRC Press, Boca Raton, 2016).

[3] T. J. Kippenberg, and K. J. Vahala, Science 321, 1172 (2008).

[4] V. B. Braginsky, Zhurnal Eksperimental'noy Teoreticheskoy Fiziki 53, 1434 (1967).

[5] C. K. Law, Phys. Rev. A 51, 2537 (1995).

[6] S. P. Kumar and M. B. Plenio, Phys. Rev. A 97, 063855 (2018).

[7] K. Sala, and T. Tufarelli, Scientific reports 8, 157 (2018).

[8] D. E. Bruschi, Journal of Mathematical Physics 61, 032102 (2020).

[9] M. G. Paris, International Journal of Quantum Information 7, 125 (2009).

[10] C. W. Helstrom, Journal of Statistical Physics 1, 231 (1969).

[11] J. Z. Bernád, C. Sanavio, and A. Xuereb, Phys. Rev. A, 97, 063821 (2018).

[12] D. Šafránek, Journal of Physics A: Mathematical and Theoretical 52, 035304 (2018).

[13] M. Szczykulska, T. Baumgratz, and A. Datta, Advances in Physics: X 1, 621-639 (2016).

[14] G. Adesso, S. Ragy, and A. R. Lee, Open Systems \& Information Dynamics 21, 1440001 (2014).

[15] D. Braun, G. Adesso, F. Benatti, R. Floreanini, U. Marzolino, M. W. Mitchell, and S. Pirandola, 2018, Rev. Mod. Phys. 90, 035006 (2018).

[16] A. Serafini, Quantum Continuous Variables: A Primer of Theoretical Methods (CRC Press, Boca Raton, 2017).
[17] C. Sanavio, J. Z. Bernád, and A. Xuereb, Phys. Rev. A 102, 013508 (2020).

[18] F. Schneiter, S. Qvarfort, A. Serafini, A. Xuereb, D. Braun, D. Rätzel, and D. E. Bruschi, Phys. Rev. A 101, 033834 (2020).

[19] C. Ventura-Velázquez, B. M. Rodríguez-Lara, H. M. Moya-Cessa, Physica Scripta 90, 068010 (2015).

[20] I. Wilson-Rae, N. Nooshi, J. Dobrindt, T. J. Kippenberg and W. Zwerger, New J. Phys. 10, 095007 (2008)

[21] C. A. Brasil, F. F. Fanchini, and R. D. J. Napolitano, Revista Brasileira de Ensino de Física 35, 01 (2013).

[22] H. Breuer, and F. Petruccione, The Theory of Open Quantum Systems (Oxford University Press, Oxford, 2010).

[23] V. Giovannetti, and D. Vitali, 2001, Phys. Rev. A 63, 023812 (2001).

[24] A. Monras, arXiv:1303.3682 (2013).

[25] M. Zwierz, C. A. Perez-Delgado, and P. Kok, Phys. Rev. Lett. 105, 180402 (2010).

[26] S. Zhou, C. L. Zou, and L. Jiang, Quantum Sci. Technol. 5025005 (2020).

[27] A. A. Berni, T. Gehring, B. M. Nielsen, V. Händchen, M. G. Paris, and U. L. Andersen, Nature Photonics 9, 577 (2015).

[28] C. Genes, A. Mari, D. Vitali, and P. Tombesi, Quantum Effects in Optomechanical Systems. Advances In Atomic, Molecular, and Optical Physics 57, 33 (2009) Academic Press,

[29] A. Luati, The Annals of Statistics 32, 1770 (2014).

[30] E. L. Lehmann, and G. Casella, Theory of point estimation (Springer-Verlag, New York, 1998). 
[31] K. Nakamura, and M. K. Fujimoto, M.K, arXiv:1711.03713 (2017).

[32] J. D. Thompson, B. M. Zwickl, A. M. Jayich, F. Marquardt, S. M. Girvin, and J. G. E. Harris, Nature 452, 72 (2008).

[33] J. D. Teufel, T. Donner, D. Li, J. W. Harlow, M. S. Allman, K. Cicak, A. J. Sirois, J. D. Whittaker, K. W. Lehnert, and R. W. Simmonds, Nature 475, 359 (2011).

[34] M. Brunelli, O. Houhou, D. W. Moore, A. Nun- nenkamp, M. Paternostro, and A. Ferraro, Phys. Rev. A 98, 063801 (2018).

[35] J. Eisert, and M. B. Plenio, International Journal of Quantum Information 1, 479-506 (2003).

[36] M. G. Genoni, and M. G. Paris, Phys. Rev. A 82, 052341 (2010).

[37] L. Latmiral, F. Armata, M. G. Genoni, I. Pikovski, and M. S. Kim Phys. Rev. A 93, 052306 (2016). 